\title{
NLRC5 regulates MHC class I antigen presentation in host defense against intracellular pathogens
}

Yikun Yao ${ }^{1, *}$, Yalong Wang ${ }^{1, *}$, Fuxiang $\mathrm{Chen}^{2}$, Yin Huang ${ }^{1}$, Shu Zhu ${ }^{1}$, Qibin Leng ${ }^{3}$, Hongyan Wang ${ }^{4}$, Yufang Shi $^{1}$, Youcun Qian

${ }^{I}$ The Key Laboratory of Stem Cell Biology, Institute of Health Sciences, Shanghai Institutes for Biological Sciences, Chinese Academy of Sciences/Shanghai Jiao Tong University School of Medicine, Shanghai 200025, China; ${ }^{2}$ Laboratory of Clinical Immunology, the Ninth People's Hospital, School of Medicine, Shanghai Jiao Tong University, Shanghai 200011, China; ${ }^{3}$ Key Laboratory of Molecular Virology and Immunology, Institut Pasteur of Shanghai, Chinese Academy of Sciences, Shanghai 200025, China; ${ }^{4}$ The State Key Laboratory of Cell Biology, Institute of Biochemistry and Cell Biology, Shanghai Institutes for Biological Sciences, Chinese Academy of Sciences, Shanghai 200031, China

NOD-like receptors (NLRs) are a family of intracellular proteins that play critical roles in innate immunity against microbial infection. NLRC5, the largest member of the NLR family, has recently attracted much attention. However, in vitro studies have reported inconsistent results about the roles of NLRC5 in host defense and in regulating immune signaling pathways. The in vivo function of NLRC5 remains unknown. Here, we report that NLRC5 is a critical regulator of host defense against intracellular pathogens in vivo. NLRC5 was specifically required for the expression of genes involved in MHC class I antigen presentation. NLRC5-deficient mice showed a profound defect in the expression of MHC class I genes and a concomitant failure to activate $L$. monocytogenes-specific $\mathrm{CDB}^{+} \mathrm{T}$ cell responses, including activation, proliferation and cytotoxicity, and the mutant mice were more susceptible to the pathogen infection. NLRP3-mediated inflammasome activation was also partially impaired in NLRC5-deficient mice. However, NLRC5 was dispensable for pathogen-induced expression of NF-кB-dependent pro-inflammatory genes as well as type I interferon genes. Thus, NLRC5 critically regulates MHC class I antigen presentation to control intracellular pathogen infection.

Keywords: NLRC5; MHC class I; infection; inflammasome

Cell Research (2012) 22:836-847. doi:10.1038/cr.2012.56; published online 10 April 2012

\section{Introduction}

NOD-like receptors (NLRs) are a recently identified family of intracellular proteins [1-5]. NLRs typically contain three structural domains: an N-terminal effectorbinding region, such as a caspase recruitment domain (CARD), pyrin domain (PYD) or baculovirus inhibitor repeat (BIR) domain; a central NBD/NACHT domain, which is essential for nucleotide binding and self-oligomerization; and a C-terminal leucine-rich repeat (LRR),

\footnotetext{
*These two authors contributed equally to this work.

Correspondence: Youcun Qian

Tel: +86-21-63852804; Fax: +86-21-63848507

E-mail: ycqian@sibs.ac.cn

Received 4 March 2012; revised 15 March 2012; accepted 16 March 2012; published online 10 April 2012
}

which is thought to detect conserved microbial patterns. Some NLRs play critical roles in directly sensing intracellular pathogens; these include Nod1, Nod2 and IPAF [2-4]. NLRP3, one of the best-characterized NLRs, is critical in sensing a variety of pathogens, such as bacterial and viral infections, as well as sterile danger signals, such as alum and monosodium urate (MSU), although NLRP3 may sense these signals indirectly $[1,5,6]$. One main feature of NLRP3 activation is the formation of the inflammasome complex consisting of NLRP3, the adaptor ASC and caspase-1. Activation of the inflammasome complex results in caspase-1 activation and consequent IL- $1 \beta$ maturation. Bioinformatics analyses reveal that there are at least 22 NLRs in humans and at least 34 NLRs in mice [2]. However, the functions of many NLRs are still unknown.

NLRC5 is the largest NLR member because of its un- 
usually long stretch of C-terminal LRRs, and it is highly conserved during vertebrate evolution [7]. Over the past two years, several in vitro studies reported the roles of NLRC5 in immune regulation and host defense [8,9]. NLRC 5 can be upregulated by IFN- $\gamma$ and bacterial or viral pathogens $[3,10,11]$. NLRC5 was originally found to negatively regulate the NF- $\mathrm{kB}$ and type I interferonproducing signaling pathways by targeting the IKK complex and RIG-I/MDA5, respectively [12]. A subsequent report showed that NLRC5 suppressed LPS-mediated inflammatory responses [13]. However, two other studies reported the contradictory result that NLRC5 promoted type I interferon production to potentiate anti-viral responses $[10,14]$. Interestingly, an in vitro study showed that NLRC5 associated with the promoters of MHC class I genes and regulated their expression in human cell lines in vitro [11]. Further analyses showed that the nucleotide-binding domain of NLRC5 was critical for its nuclear import and transactivation activity [15]. NLRC5 was also found to contribute to NLRP3 inflammasome activation in vitro [16]. However, one recent in vivo study did not find any role for NLRC5 [17]. Therefore, the roles of NLRC5 remain unclear. We generated NLRC5-deficient mice and found that NLRC5 was required for host defense against intracellular pathogen infection. NLRC5 critically regulated MHC class I gene expression and $\mathrm{CD} 8^{+} \mathrm{T}$ cell activation, proliferation and cytotoxicity. NLRC5 also partially regulated NLRP3 inflammasome activation. Our study therefore is the first to demonstrate the in vivo functions of NLRC5.

\section{Results}

NLRC5 regulates the expression of genes involved in MHC class I antigen presentation

To study the in vivo function of NLRC5, we generated NLRC5-deficient $\left(\mathrm{NlrC}^{-/-}\right)$mice (Supplementary information, Figure S1). Genotyping of the $N \operatorname{lrc} 5^{-/-}$mice showed that NLRC5 mRNA was successfully depleted (Supplementary information, Figure S1). The $\mathrm{Nlrc}^{-/-}$ mice were born normally at the expected Mendelian frequency (data not shown). Consistent with a previous report [11], we observed both cytoplasmic and nuclear localization of NLRC5, and mutation of the nuclear localization signal in NLRC5 resulted in the disappearance of its nuclear localization (data not shown), suggesting that NLRC5 may have a role in the nucleus. Interestingly, NLRC5 has been reported to regulate MHC class I gene expression in a human cell line in vitro [11]. To determine whether NLRC5 regulates the expression of MHC class I genes in vivo, we examined the mRNA expression of MHC class Ia genes (H2D and H2K) and Ib genes
(H2M3 and H2Qa1) and found that their expression was reduced in the NLRC5-deficient spleen (Figure 1A) and thymus (Supplementary information, Figure S2A). The mRNA expression of the genes involved in MHC class I antigen presentation and processing, including B2M, LMP2 and TAP1, was also reduced in the NLRC5-deficient spleen (Figure 1A) and thymus (Supplementary information, Figure S2A), whereas the mRNA expression of MHC class II genes, including $\mathrm{A} \alpha, \mathrm{E} \beta$ and $\mathrm{O} \alpha$, was not significantly altered under NLRC5 deficiency (Figure $1 \mathrm{~A}$ and Supplementary information, Figure S2A). These data suggest that NLRC5 specifically regulates genes for MHC class I antigen presentation. H2K protein expression, as determined by flow cytometry analysis, was similarly reduced in the NLRC5-deficient spleen, liver, lymph nodes and thymus (Supplementary information, Figure S2B). We then investigated whether NLRC5 regulates MHC class I gene expression in mouse cells in vitro. Whereas LPS induced the mRNA expression of the MHC class I genes and the genes involved in MHC class I antigen presentation and processing in wild-type bone marrow macrophages (BMMs), the induction of these genes was significantly reduced in NLRC5-deficient cells (Figure 1B). LPS also induced NLRC5 expression (Figure 1B), indicating that NLRC5 is required for the induction of the genes for MHC class I antigen presentation. As MHC class I antigen presentation is critical for host defense against intracellular pathogens, we next examined whether NLRC5 regulates MHC class I gene expression during infection. We chose the typical intracellular pathogen $L$. monocytogenes (LM) to infect mice and found that the pathogen induced the expression of NLRC5 (Figure 1C). Whereas the intracellular pathogen induced the expression of the genes involved in MHC class I antigen presentation in wild-type mice, the induction of those genes was severely impaired in NLRC5-deficient mice (Figure 1C). As a control, the expression of MHC class II genes was not dramatically induced by LM (Figure 1C). The induction of $\mathrm{H} 2 \mathrm{~K}$ at the protein level was also reduced in NLRC5-deficient mice during the pathogen infection (Supplementary information, Figure S2C). Together, our data show that NLRC5 specifically regulates the expression of the genes for MHC class I antigen presentation both in vitro and in vivo.

\section{NLRC5 regulates $C D 8^{+} T$ cell activation to constrain $L M$ infection}

MHC class I antigen presentation is critical for $\mathrm{CD}^{+}$ $\mathrm{T}$ cell development and activation $[18,19]$. We therefore evaluated T cell development in NLRC5-deficient mice. Whereas $\mathrm{CD}^{+}$cell development was normal, the $\mathrm{CD} 8^{+}$ $\mathrm{T}$ cell number was significantly reduced in the spleen 
A
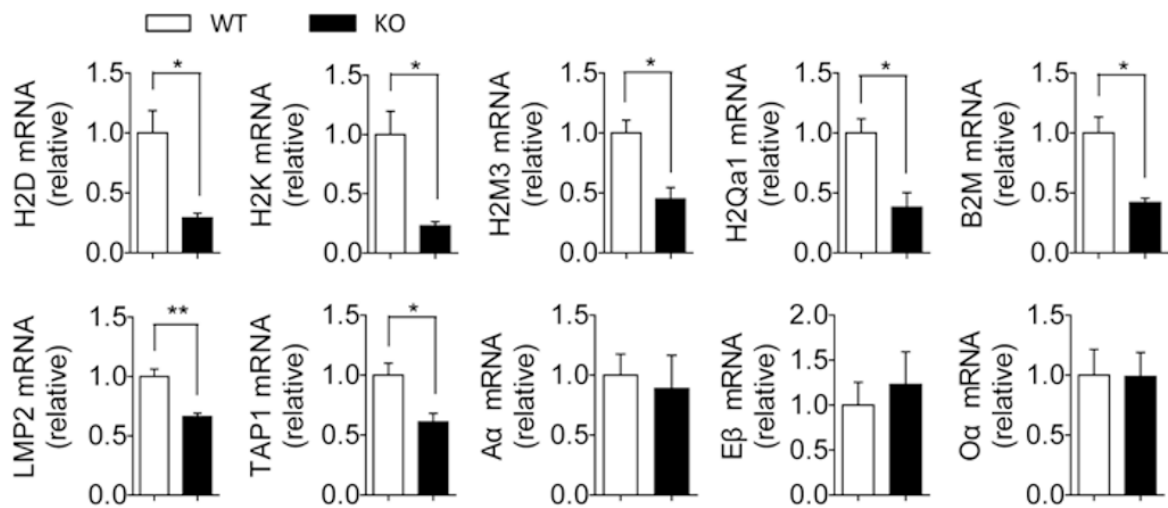

B
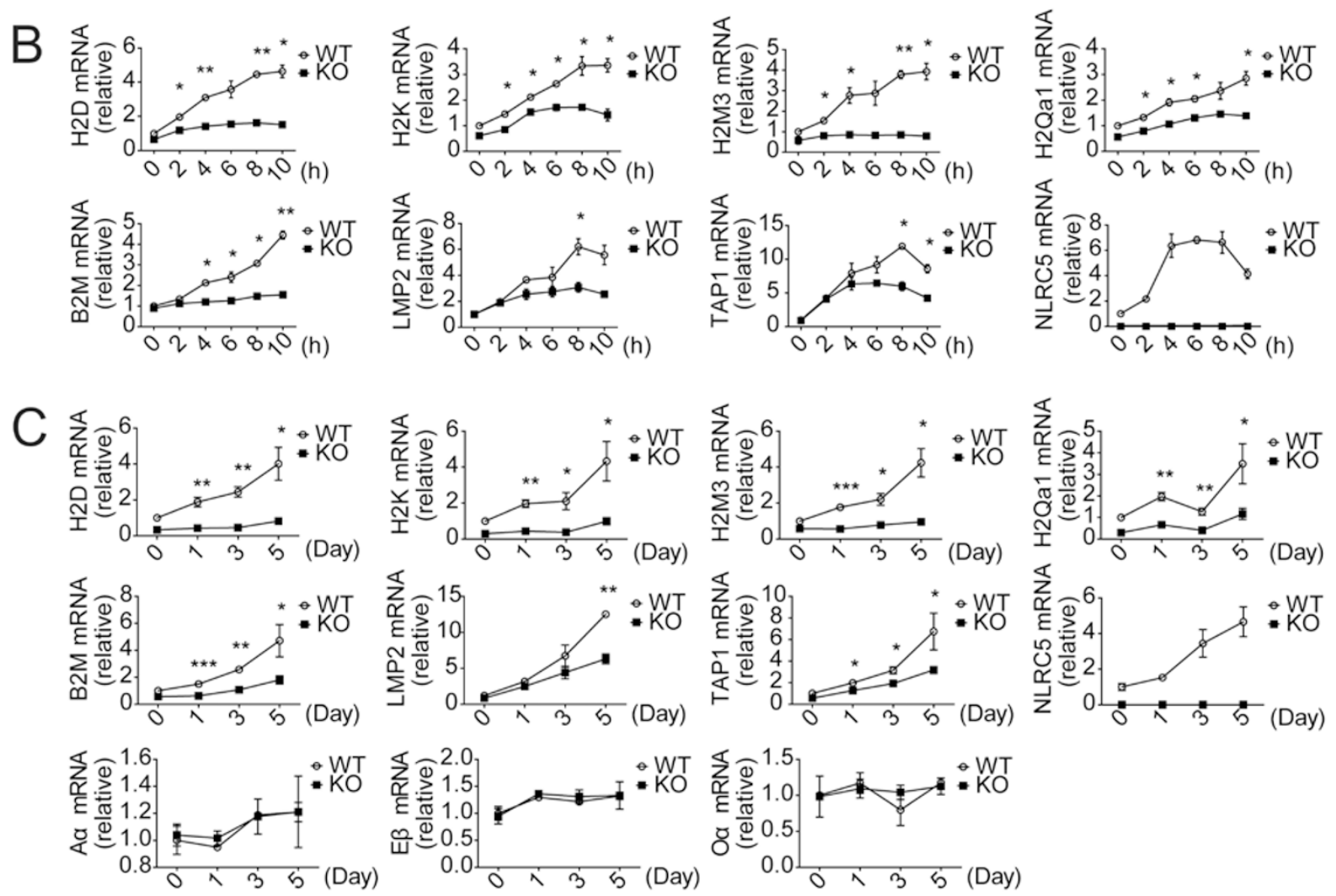

Figure 1 NLRC5 specifically regulates the expression of genes involved in MHC class I antigen presentation. (A) Quantitative RT-PCR analysis of MHC class la genes (H2D and H2K), MHC class lb genes (H2M3 and H2Qa1), MHC class I functionally relative genes (B2M, LMP2 and TAP1) and MHC class II genes $(A \alpha, B \beta, O \alpha)$ in the spleen tissues from N/rc $5^{+/+}$(WT) and NIrc5 ${ }^{-1-}(\mathrm{KO})$ mice. (B) Quantitative RT-PCR analysis of those MHC class I genes and MHC class I-related genes as indicated in $\mathbf{A}$ in bone marrow-derived macrophages from $\mathrm{NIrC5}^{+/+}$(WT) and $\mathrm{Nrc5}^{-/-}$(KO) mice that were unstimulated or stimulated with LPS (100 ng/ml) for the indicated times. (C) Quantitative RT-PCR analysis of the genes indicated in B in the spleen tissues from N/rc5 $5^{+/}(\mathrm{WT})$ and $\mathrm{NIrc5}^{-1-}(\mathrm{KO})$ mice infected i.v. with $2 \times 10^{4}$ colony-forming units (CFU) of LM for the indicated times. ${ }^{*} P<0.05 ;{ }^{* *} P<0.01 ;{ }^{* \star \star} P<0.001$ (Student's $t$-test). Data represent three independent experiments $(n=4$; mean \pm SEM in A-C).

of NLRC5-deficient mice (Supplementary information, Figure S3A). The cell numbers of CD4 and CD8 doublepositive, single-positive or double-negative $\mathrm{T}$ cells were not significantly changed in the NLRC5-deficient thymus
(Supplementary information, Figure S3B), consistent with a previous report that these cell numbers in thymus are not affected by a deficiency in genes involved in MHC class I antigen presentation [18]. As NLRC5 regu- 
lated the expression of the genes involved in MHC class I antigen presentation during LM infection (Figure 1C), we examined $\mathrm{CD}^{+} \mathrm{T}$ cells at day 7 after the pathogen infection, at which time an adaptive immune response should have developed. Indeed, the percentage of CD $8^{+}$ $\mathrm{T}$ cells was dramatically reduced, whereas the $\mathrm{CD} 4^{+} \mathrm{T}$ cell percentage was not significantly altered in the spleen or liver of NLRC5-deficient mice compared to their wild-type littermates (Figure 2A-2D). Further analyses showed that the total number of $\mathrm{CD}^{+} \mathrm{T}$ cells was dramatically decreased, but the total number of $\mathrm{CD}^{+} \mathrm{T}$ cells was not significantly altered in the spleen (Figure 2E) or liver (Figure 2F) of NLRC5-deficient mice both at day 3 and at day 7 after LM infection.

As NLRC5 regulated the expression of the genes involved in MHC class I antigen presentation during infection, the reduced number of $\mathrm{CD} 8^{+} \mathrm{T}$ cells in NLRC5deficient mice could be due to impaired $\mathrm{CD} 8^{+} \mathrm{T}$ cell activation. To address whether this was the case, double staining for $\mathrm{CD} 8$ and IFN- $\gamma$ was utilized to detect $\mathrm{CD} 8^{+} \mathrm{T}$ cell activation by flow cytometry. Indeed, the percentage or total number of $\mathrm{CD}^{+} \mathrm{IFN}^{-} \gamma^{+}$cells was dramatically reduced in the spleen and liver of NLRC5-deficient mice compared to wild-type control mice (Figure $2 \mathrm{G}$ and $2 \mathrm{H}$ ), whereas the percentage or total number of $\mathrm{CD} 4^{+} \mathrm{IFN}-\gamma^{+}$ $\mathrm{T}$ cells was not significantly changed (Supplementary information, Figure S3C and S3D), consistent with the specific regulation of MHC class I genes but not class II genes by NLRC5. ELISAs also showed significantly reduced IFN- $\gamma$ production in the NLRC5-deficient spleen and liver after infection (Figure 2I). To determine whether NLRC5 deficiency in $\mathrm{CD} 8^{+} \mathrm{T}$ cells also affected their activation capacity, $\mathrm{CD} 8^{+} \mathrm{T}$ cells from wild-type or NLRC5-deficient mice were purified and stimulated with anti-CD3 plus anti-CD28 in vitro. TCR activation-induced IFN- $\gamma$ production was normal in NLRC5-deficient $\mathrm{CD}^{+} \mathrm{T}$ cells (Supplementary information, Figure S4A). TCR signaling was also not affected in NLRC5-deficient $\mathrm{CD}^{+} \mathrm{T}$ cells (Supplementary information, Figure S4B). These results indicate that there is no intrinsic defect in NLRC5-deficient $\mathrm{CD} 8^{+} \mathrm{T}$ cells and that the impaired $\mathrm{CD}^{+} \mathrm{T}$ cell activation in NLRC5-deficient mice in vivo is due to the reduced levels of MHC class I gene expression. As NLRC5 regulates MHC class I-mediated CD8 ${ }^{+}$ $\mathrm{T}$ cell activation, it may affect host defense against intracellular pathogens. Indeed, the bacterial titer of LM was significantly increased in the spleen (Figure 2J) and liver (Figure 2K) of NLRC5-deficient mice compared to wildtype controls, indicating that NLRC5 is required for host defense against intracellular pathogen infections. Together, our data suggest that NLRC5 specifically regulates the expression of the genes involved in MHC class I antigen presentation for subsequent $\mathrm{CD} 8^{+} \mathrm{T}$ cell activation to kill intracellular pathogens.

NLRC5 regulates $M H C$ class I-mediated $C D 8^{+} T$ cell activation, proliferation and cytotoxicity

We showed above that NLRC5 regulates MHC class I gene expression and $\mathrm{CD}^{+} \mathrm{T}$ cell activation during infection. To further confirm whether NLRC5 regulates antigen-specific $\mathrm{T}$ cell activation, naive $\mathrm{OT}-1 \mathrm{CD} 8^{+} \mathrm{T}$ cells, which carry a TCR that recognizes OVA peptide, were mixed with either wild-type or NLRC5-deficient bone marrow-derived dendritic cells (BMDCs) that had been pulsed with OVA peptide. OVA-induced production of IFN- $\gamma$ was significantly reduced under NLRC5 deficiency (Figure 3A), indicating that NLRC5 expressed in BMDCs is important for antigen-specific $\mathrm{CD} 8^{+} \mathrm{T}$ cell activation. CFSE (carboxyfluorescein succinimidyl ester) staining analyses showed that NLRC5 deficiency impaired OVA-induced OT-1T cell proliferation (Figure $3 \mathrm{~B}$ ), indicating that NLRC5 in BMDCs is also important for antigen-specific $\mathrm{CD} 8^{+} \mathrm{T}$ cell proliferation. To determine whether NLRC5 regulates MHC class I antigen presentation in target cells that could be lysed by antigen-activated $\mathrm{CD} 8^{+} \mathrm{T}$ cells, BMDCs from NLRC5deficient mice or wild-type control mice were pulsed with OVA and then used as target cells by mixing them with the same antigen-activated OT-1 T cells in vitro. The cytolysis results show that activated OT-1 T cells killed far fewer OVA-pulsed NLRC5-deficient target cells, indicating that $\mathrm{MHC}$ class I antigen presentation was impaired in NLRC5-deficient cells (Figure 3C). To further determine whether NLRC5 regulated antigenspecific $\mathrm{CD}^{+} \mathrm{T}$ cell cytotoxicity, NLRC5-deficient mice or wild-type mice were infected with OVA-expressing LM, and the mice were then injected intravenously (i.v.) with a mixture of OVA-pulsed and -unpulsed splenocytes. The OVA-specific cytolysis was significantly reduced in NLRC5-deficient spleens (Figure 3D and 3E) and livers (Figure 3D and 3F). Together, our data suggest that NLRC5 regulates antigen-specific $\mathrm{CD} 8^{+} \mathrm{T}$ cell activation, proliferation and cytotoxicity.

\section{NLRC5 promotes inflammasome activation}

Some NLR members, such as NLRP3, activate the inflammasome [5]. To determine whether NLRC5 is involved in inflammasome activation, NLRC5 or a mutant with the LRR domain deleted, a mutation that is designed to remove the autoinhibitory effect of LRRs, was overexpressed to determine the effect on the processing of procaspase- 1 and pro-IL-1 $\beta$. Overexpressed NLRC 5 or its deletion mutant activated this processing, and the mutant also promoted NLRP3 inflammasome activation (Sup- 
plementary information, Figure S5A), consistent with recent reports $[16,17]$. Also as reported previously [16], NLRC5 associated with NLRP3 (Supplementary infor- mation, Figure S5B), and the NACHT domain of NLRC5 mediated this association with NLRP3 (Supplementary information, Figure S5C). We further found that NLRC5

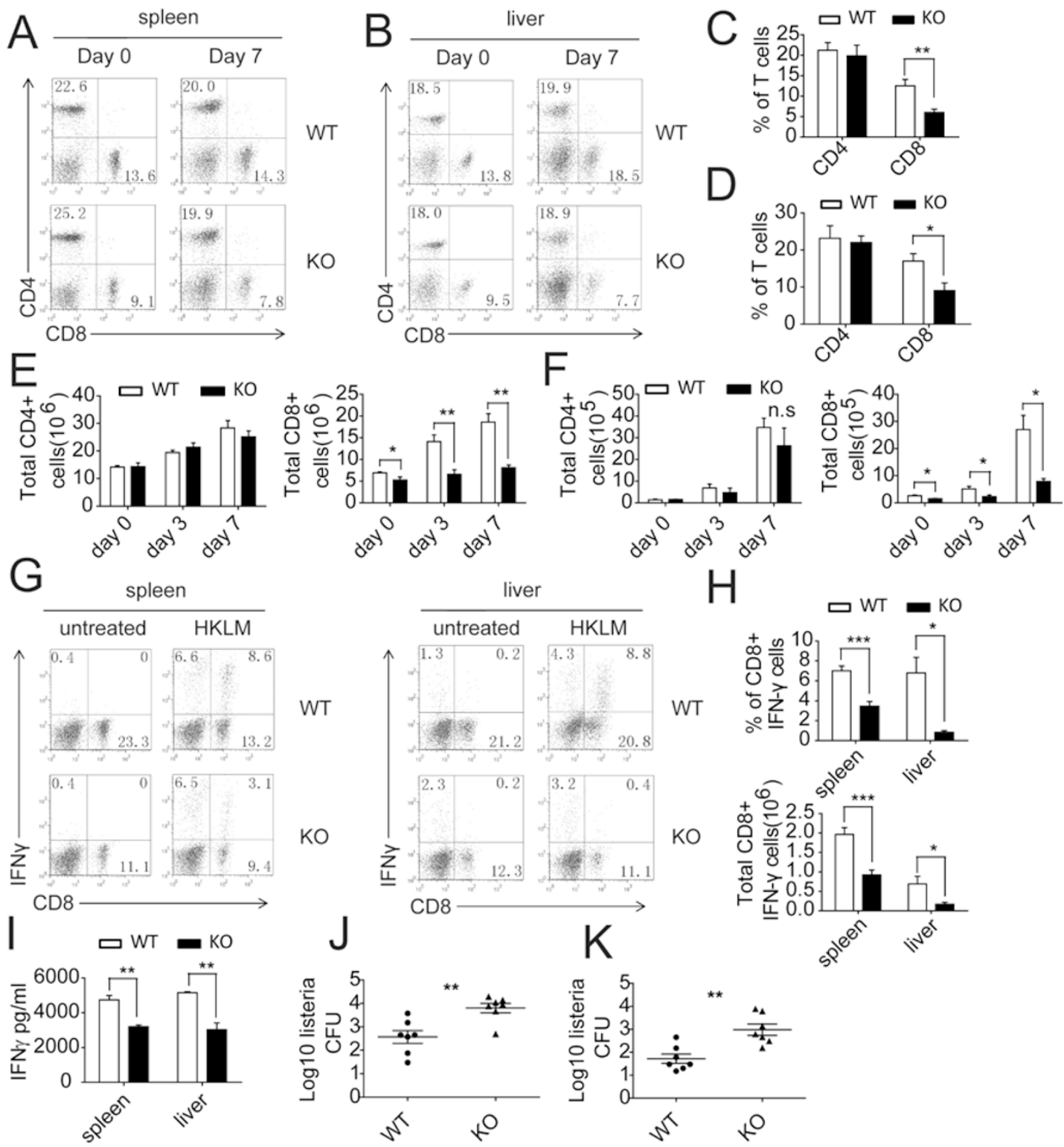

Figure 2 NLRC5 regulates CD8 ${ }^{+}$T cell activation to constrain LM infection. (A, B) Splenocytes (A) or hepatic leukocytes (B) were harvested from NIrc5 $5^{+/+}(\mathrm{WT})$ and $\operatorname{NIrc5}^{-1}(\mathrm{KO})$ mice infected i.v. with LM $\left(2 \times 10^{4} \mathrm{CFU}\right)$ for 7 days, and stained with antibodies against CD8 $\alpha$-FITC and CD4-PE for flow cytometry analysis. Numbers in the quadrant indicate the percentage of cells. (C, D) Bar graphs represent the percentage of $C D 4^{+}$or $C D 8^{+} T$ cells in the spleen (C) or liver (D). (E, F) Splenocytes (E) or hepatic leukocytes (F) from WT and $\mathrm{NIrc5}^{-/-}(\mathrm{KO})$ mice infected as in A for the indicated times were analyzed by flow cytometry for the total number of $\mathrm{CD}^{+}$or $\mathrm{CD}^{+} \mathrm{T}$ cells per spleen (E) or per liver (F). (G) Splenocytes or hepatic leukocytes isolated from WT and $\mathrm{NIrC5}^{-1-}$ (KO) mice infected as in A were untreated or stimulated with HKLM overnight. Cells were then stained with antibodies against CD $8 \alpha-\mathrm{FITC}$ and IFN- $\gamma-\mathrm{PE}$ and analyzed by flow cytometry. Numbers in the quadrant indicate the percentage of cells. (H) Bar graphs represent the percentage or total number of $\mathrm{CD} 8^{+} \mathrm{IFN}-\gamma^{+} \mathrm{T}$ cells. (I) IFN- $\gamma$ level in the supernatant of the cultured cells as in $\mathbf{G}$ was measured by ELISA. $(\mathbf{J}, \mathbf{K})$ Bacterial burden was determined in the spleens (J) and livers (K) of WT and NIrc5 ${ }^{-1-}(\mathrm{KO})$ mice infected as in A. ${ }^{*} P<0.05$; ${ }^{* \star} P<0.01$; ${ }^{* *} P<0.001$ (Student's $t$-test). Data are representative of three $(\mathbf{A}-\mathbf{B}, \mathbf{J}-\mathbf{K})$ or two $(\mathbf{C}-\mathbf{F}, \mathbf{H}-\mathbf{I})$ independent experiments $(n=4$; mean \pm SEM in $\mathbf{C}-\mathbf{F}, \mathbf{H}-\mathbf{K})$. 
A

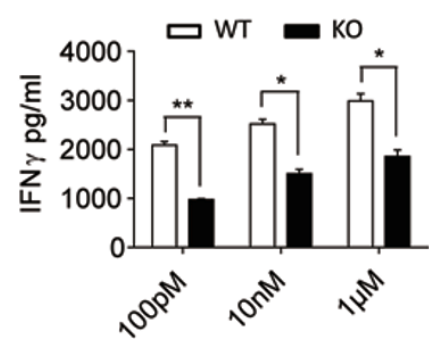

OVA
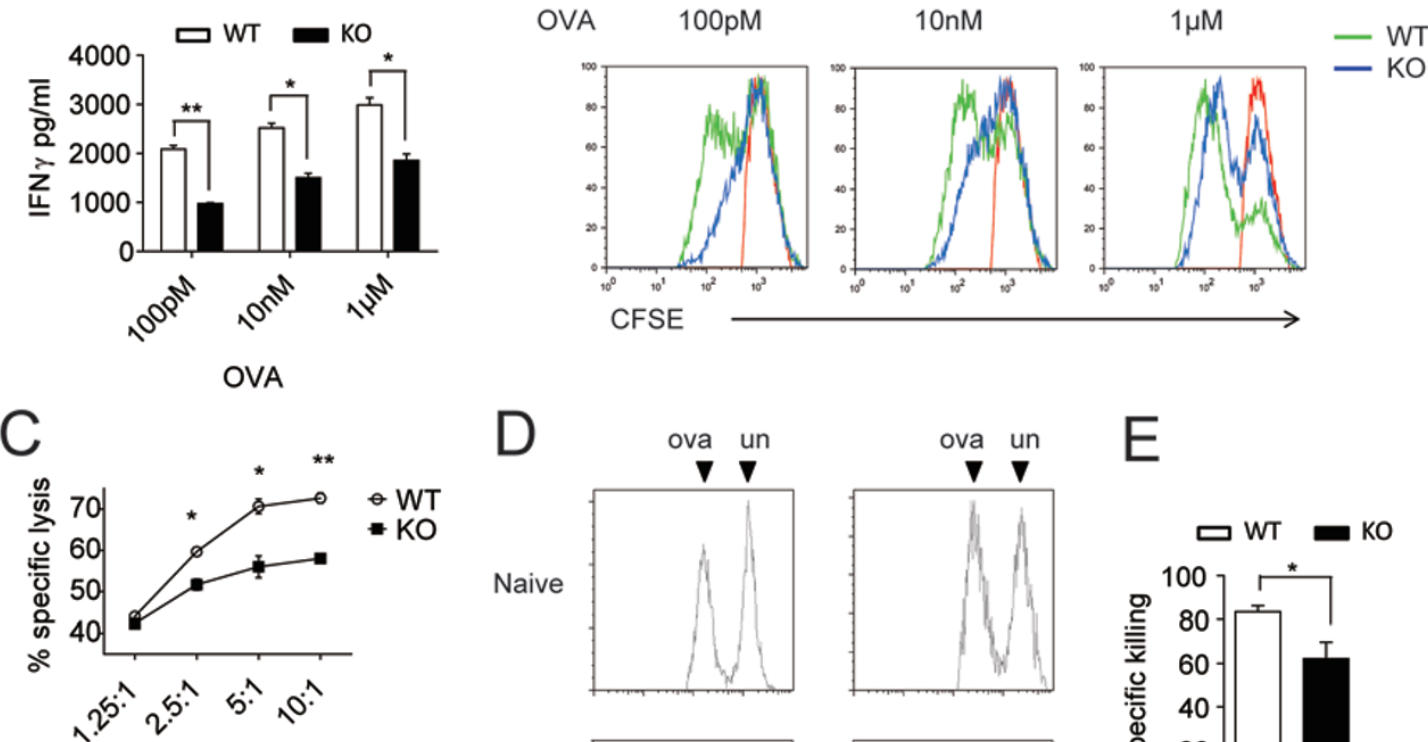

\section{E:T}
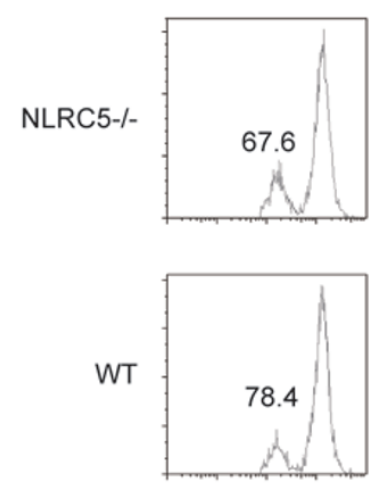

spleen
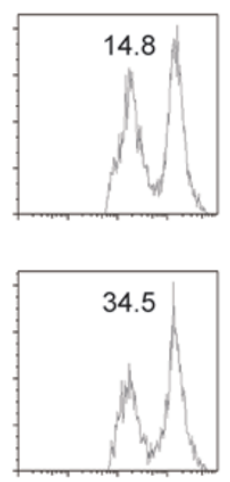

liver
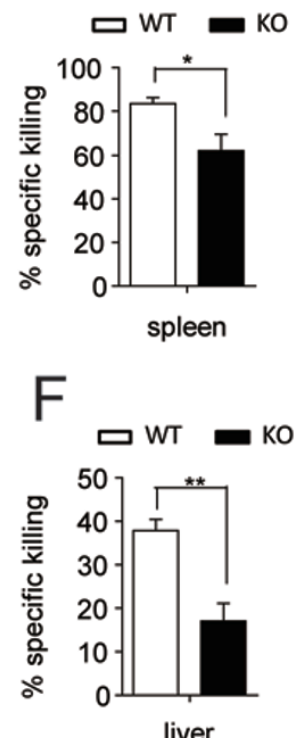

Figure 3 NLRC5 regulates MHC class I-mediated CD8 ${ }^{+}$T cell activation, proliferation and cytotoxicity. (A, B) Purified naive OT-1 CD8 ${ }^{+}$T cells labeled with $4 \mu \mathrm{M}$ CFSE were cultured with WT or NIrc5 ${ }^{-1}$ (KO) BMDCs that had been pulsed with OVA at a series of concentrations (from $100 \mathrm{pM}$ to $1 \mu \mathrm{M}$ ) at a ratio of 3:1 in plate-bound assays for 3 days. IFN- $\gamma$ levels in the supernatant were determined by ELISA and represent T cell activation (A). CFSE intensity in T cells was determined by flow cytometry, representing OT-1 CD8 ${ }^{+} \mathrm{T}$ cell proliferation (B). (C) OT-1 T cells were treated with OVA peptide and used as effector cells. BMDCs from WT or NIrc $5^{-1-}$ mice were pulsed with OVA (100 pM) for $1 \mathrm{~h}$ at $37^{\circ} \mathrm{C}$ and used as target cells. The effector and target cells were co-cultured at the ratios indicated. Cytotoxic T lymphocyte-mediated killing was determined by LDH release using a cytotoxicity detection kit. (D) C57BL/6 splenocytes labeled with CFSE were untreated (un) or OVA-pulsed (ova), then co-injected i.v. into naive mice or $\mathrm{NIrC5}^{-1}$ and wild-type (WT) control mice that had been infected with LM-OVA (2 $\times 10^{4}$ CFU) for three days. In vivo cytolysis was assessed 16 h later by flow cytometry. Histograms were gated on CFSE-positive target cells, and the numbers above each peak represent the percent of specific killing, which was calculated as described in Materials and Methods. (E, F) Bar graphs indicate the percentage of specific lysis in the spleen (E) or liver (F) as analyzed in $\mathbf{D}$. ${ }^{*} P<0.05 ;{ }^{* *} P<0.01$ (Student's $t$-test). Data are representative of three $(\mathbf{A}-\mathbf{C}, n=3)$ or two $(\mathbf{D}-\mathbf{F}, n=4)$ independent experiments (mean \pm SEM in A, C, E, F).

interacted with the inflammasome complex components ASC and caspase-1 (Supplementary information, Figure S5D). Thus, NLRC5 likely contributes to inflammasome activation.

To investigate whether NLRC5 regulates the NLRP3 inflammasome in mouse cells, NLRC5-deficient BMMs were treated with the NLRP3 ligand MSU, alum or LPS plus ATP. Immunoblotting analyses showed that the MSU- or LPS plus ATP-mediated processing of procaspase-1 or pro-IL-1 $\beta$ was reduced, although not severely impaired, under NLRC5 deficiency (Figure 4A). ELISAs also showed that MSU-, alum- or LPS plus 

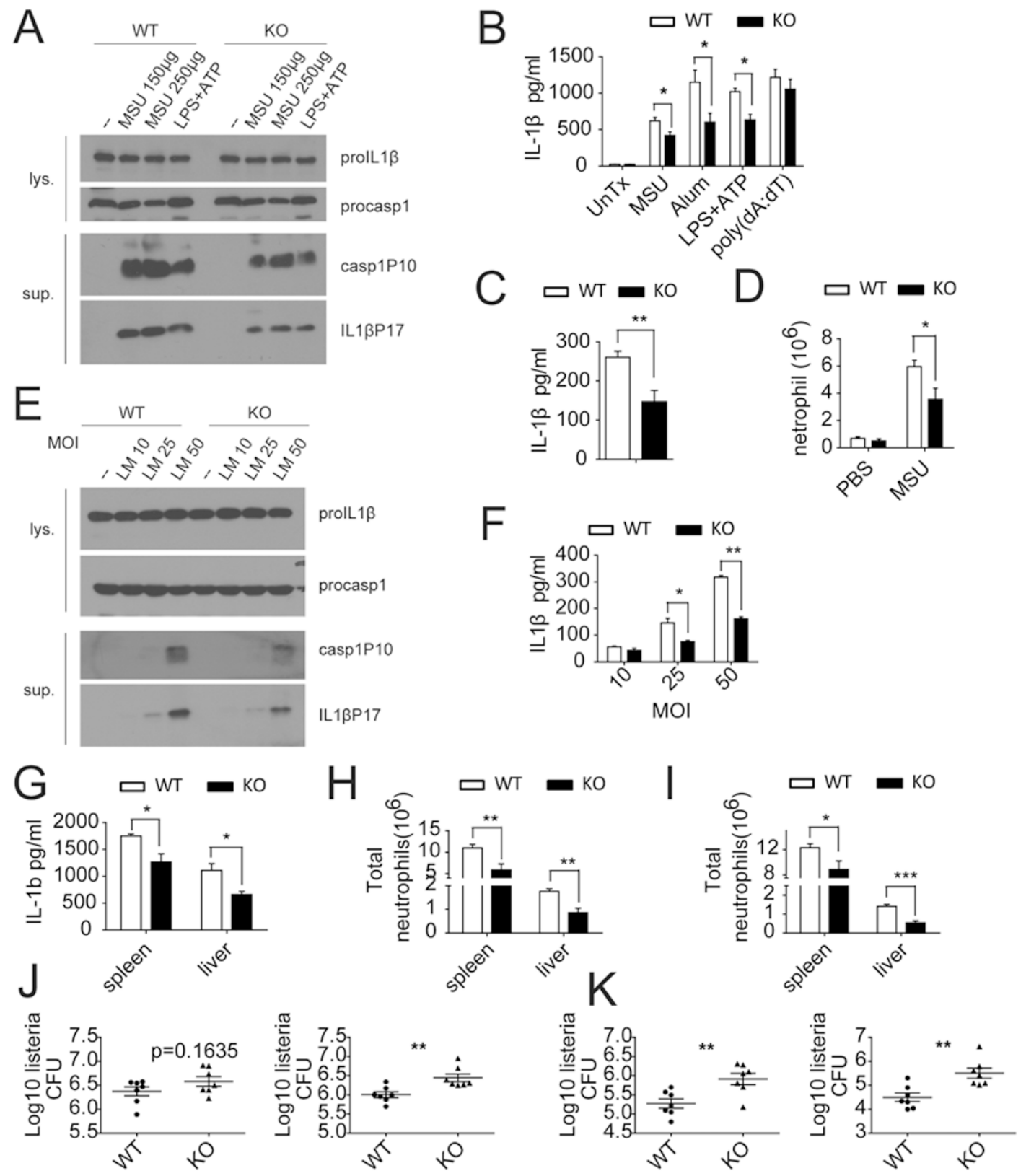

Figure 4 NLRC5 partially regulates inflammasome activation. (A, B) Bone marrow-derived macrophages (BMMs) from wildtype (WT) or N/rc5 ${ }^{-1-}(\mathrm{KO})$ mice were primed with LPS $(500 \mathrm{ng} / \mathrm{ml})$ for $6 \mathrm{~h}$ and subsequently stimulated with MSU (150 $\mu \mathrm{g} /$ $\mathrm{ml}$ or $250 \mu \mathrm{g} / \mathrm{ml})$, alum $(500 \mu \mathrm{g} / \mathrm{ml})$, LPS $(500 \mathrm{ng} / \mathrm{ml})+$ ATP $(5 \mathrm{mM})$ or poly $(\mathrm{dA}: \mathrm{dT})(1 \mu \mathrm{g} / \mathrm{ml})$ for $6 \mathrm{~h}$. Immunoblotting of cell lysates and culture supernatants was performed with the indicated antibodies for caspase- 1 and IL-1 $1 \beta$ cleavage (A). Supernatants were also analyzed for IL-1 $\beta$ by ELISA (B). (C, D) WT or N/rc $5^{-1-}$ (KO) mice were injected intraperitoneally with PBS (control) or MSU crystals (2 mg per mouse). Neutrophil influx was quantified $6 \mathrm{~h}$ later by flow cytometry (D), and supernatants of the influx were analyzed for IL-1 $\beta$ by ELISA (C). (E) BMMs from WT mice or NIrc5 ${ }^{-1-}$ (KO) mice were primed with LPS $(500 \mathrm{ng} / \mathrm{ml})$ for $3 \mathrm{~h}$ and subsequently infected with the indicated doses of LM for $30 \mathrm{~min}$. Immunoblotting of cell lysates and culture supernatants was performed with the indicated antibodies for caspase- 1 and IL-1 $\beta$ cleavage (E). Supernatants were also analyzed for IL-1 $\beta$ by ELISA (F). (G) Spleens and livers from WT and NIrc5 ${ }^{-1-}$ (KO) mice infected with LM for 1 day were homogenized, and the supernatants were evaluated for IL-1 $1 \beta$ cleavage by ELISA. (H, I) Splenocytes or hepatic leukocytes from WT and $\mathrm{NIrC5}^{-1-}(\mathrm{KO})$ mice infected with LM for 1 day $(\mathrm{H})$ or three days $(\mathrm{I})$ were stained with the antibodies anti-CD11bFITC and anti-Ly6G-PE to quantify the neutrophils by flow cytometry. (J, K) Bacterial burden was determined in the spleens and livers of WT and $\mathrm{NIrc5}^{-/-}(\mathrm{KO})$ mice infected with LM for 1 day $(\mathrm{J})$ or three days $(\mathrm{K}) .{ }^{*} P<0.05 ;{ }^{* *} P<0.01 ;{ }^{* * *} P<0.001$ (Student's $t$-test). Data are representative of three independent experiments (A-G, $n=3 ; \mathbf{H}-\mathbf{I}, n=4 ; \mathbf{J}-\mathbf{K}, n=7)($ mean \pm SEM in B-D, F-K). 
ATP-induced mature IL-1 $\beta$ secretion was significantly reduced in NLRC5-deficient cells compared to wild-type cells (Figure 4B). Although we observed that NLRC5 associated with AIM2 upon NLRC5 overexpression (data not shown), the IL-1 $\beta$ production induced by the AIM2 ligand poly(dA:dT) was not affected by NLRC5 deficiency (Figure 4B), indicating that NLRC5 is not generally involved in inflammasome activation. Further in vivo investigation showed that MSU-induced mature IL-1 $\beta$ production was significantly reduced in NLRC5-deficient mice (Figure 4C). As NLRP3-mediated IL-1 $\beta$ production is required for MSU-induced neutrophil recruitment [20], MSU-induced recruitment of neutrophils was similarly reduced in NLRC5-deficient mice (Figure 4D). These data suggest that NLRC5 promotes, but is not essential for, NLRP3 inflammasome activation.

As NLRP3 is important for LM-mediated inflammasome activation [21], we investigated whether NLRC5 contributed to the pathogen-induced IL-1 $\beta$ secretion. NLRC5 was partially required for the infectioninduced processing of pro-caspase- 1 and pro-IL-1 $\beta$ to mature caspase- 1 and IL-1 $\beta$, respectively, as determined by immunoblotting and ELISA (Figure 4E and 4F). Similarly, the infection-induced IL- $1 \beta$ secretion was partially blocked in the spleen and liver of NLRC5-deficient mice compared to wild-type mice (Figure 4G). Consistent with the reduced IL- $1 \beta$ secretion, the induced recruitment of neutrophils to the spleen and liver was significantly reduced at day 1 and day 3 after LM infection in NLRC5deficient mice compared to wild-type mice (Figure $4 \mathrm{H}$ and $4 \mathrm{I}$ ). The bacterial number was significantly increased in the spleen at day 3 and in the liver both at day 1 and at day 3 after the pathogen infection in NLRC5-deficient mice (Figure 4J and $4 \mathrm{~K}$ ), probably due to the reduced neutrophil recruitment. Together, our data suggest that NLRC5 partly regulates NLRP3 inflammasome activation, which may contribute to host innate immunity against intracellular pathogen infection through IL-1 $\beta$ mediated recruitment of neutrophils.

$N L R C 5$ regulates neither the expression of $N F-\kappa B$ dependent genes nor the expression of type I interferon genes

NLRC5 negatively regulates LPS-induced and IKKmediated NF- $\kappa \mathrm{B}$ activation as well as its downstream pro-inflammatory gene expression [12]. We examined whether NLRC5 deficiency affects the NF- $\kappa$ B activation pathway. There was no obvious difference in LPSinduced NF- $\mathrm{\kappa B}$ activation or MAPK activation between NLRC5-deficient cells and wild-type control cells (Supplementary information, Figure S6A). Similarly, LPS-induced expression of downstream genes, includ- ing IL-6, TNF $\alpha$ and IL-1 $\beta$, was not significantly altered under NLRC5 deficiency, although LPS induced NLRC5 expression (Supplementary information, Figure S6B). Similar to LPS, downstream gene expression induced by poly(I:C) and poly(dA:dT), which mimic pathogenic double-stranded RNA and DNA, respectively, was not affected by NLRC 5 deficiency, although both stimuli induced NLRC5 expression (Supplementary information, Figure S6C and S6D). Similarly, LM-induced expression of IL-6, TNF $\alpha$, IL-1 $\beta$ and IL-10 was not affected by NLRC5 deficiency, but the pathogen did induce NLRC5 expression (Figure 5A). Thus, these data suggest that NLRC5 does not regulate NF- $\mathrm{KB}$-dependent genes during pathogen infection.

NLRC5 has been reported to either negatively or positively regulate type I interferon production through targeting RIG-I or MDA5 [10, 12, 14]. To define whether NLRC5 deficiency affected IFN- $\beta$ induction, we stimulated wild-type or NLRC5-deficient bone marrowderived macrophages with poly $(\mathrm{dA}: \mathrm{dT})$ or poly $(\mathrm{I}: \mathrm{C})$ and found that NLRC5 deficiency did not affect stimulusinduced IFN- $\beta$ production (Figure $5 \mathrm{~B}$ and $5 \mathrm{C}$ ). Similarly, LM-induced expression of IFN- $\beta$ was not affected under NLRC5 deficiency (Figure 5D), although the LM induced NLRC5 expression (Figure 5A). Therefore, our results strongly suggest that NLRC5 does not regulate pathways for type I interferon production.

\section{Discussion}

The in vivo function of NLRC5 has remained unknown until recently. By generating and analyzing NLRC5-deficient mice, we showed that NLRC5 is critical for host defense against intracellular bacterial infection. NLRC5 specifically regulated the expression of genes involved in MHC class I antigen presentation and consequently, antigen-specific $\mathrm{CD} 8^{+}$activation, proliferation and cytotoxicity during infection with the intracellular pathogen LM. NLRC5 regulated IFN- $\gamma$ production through $\mathrm{MHC}$ class I-mediated $\mathrm{CD} 8^{+} \mathrm{T}$ cell activation, and IFN- $\gamma$ potently upregulated NLRC5 expression, suggesting that this positive-feedback loop is critical for hosts to efficiently kill intracellular pathogens. NLRC5 also partly regulated NLRP3 inflammasome activation during LM infection. Thus, NLRC5 regulates both innate and adaptive immunity in host defense against intracellular pathogens.

NLRC5 is found both in the cytosol and in the nucleus $[9,11,13]$. The nuclear localization signal (NLS) in NLRC5 is required for its nuclear localization [11]. Interestingly, NLRC5 is phylogenetically most closely related to CIITA, another NLR member that also contains 

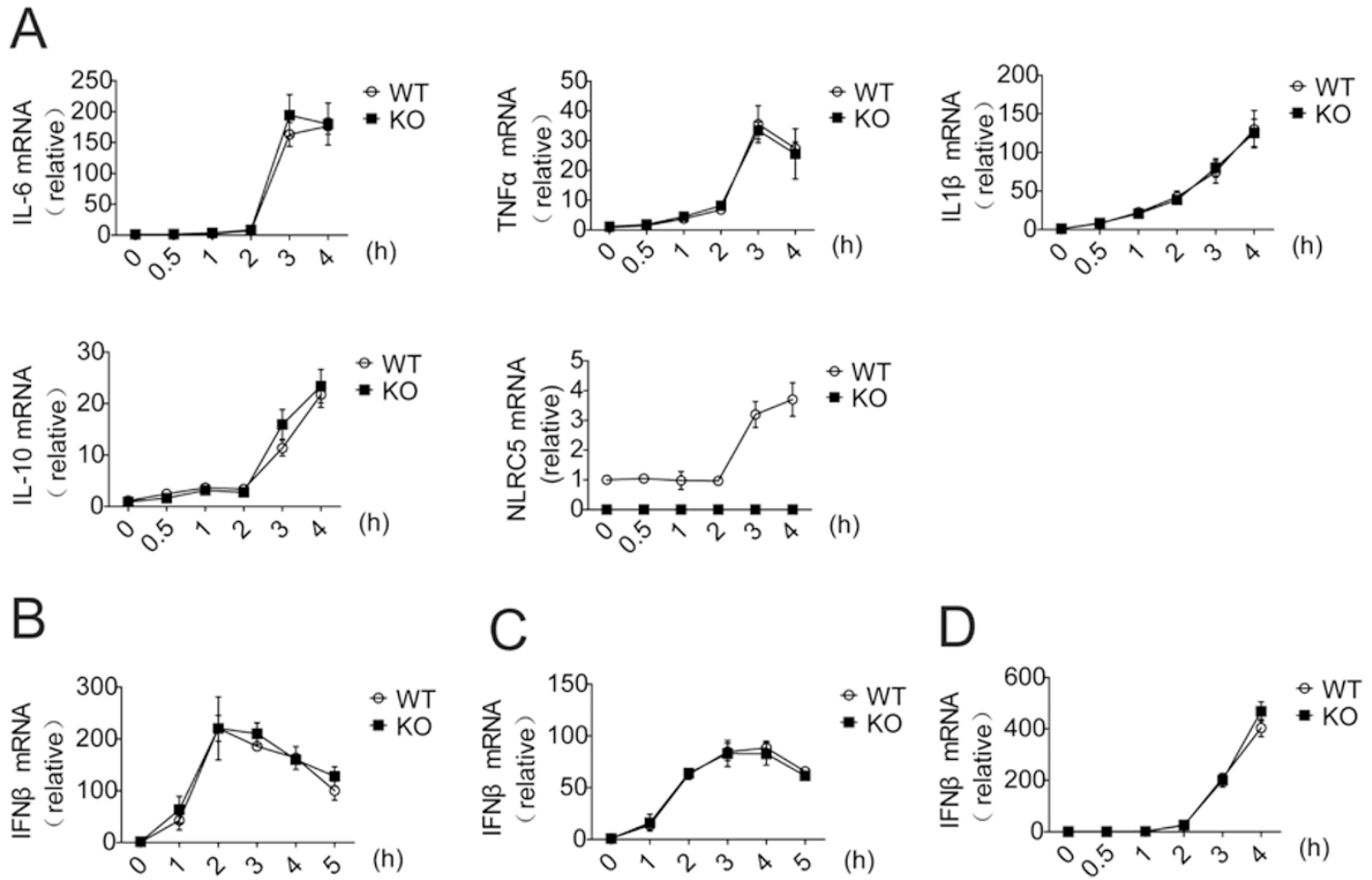

Figure 5 NLRC5 is dispensable for pathogen-induced expression of NF-kB-dependent genes and the IFN- $\beta$ gene. (A) BMMs

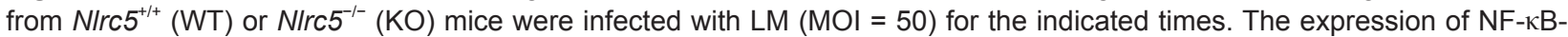
dependent genes (IL-6, TNF $\alpha$, IL-1 $\beta$, and IL-10) was determined by quantitative real-time PCR. (B, C) BMMs from NIrc5 ${ }^{+/+}(\mathrm{WT})$ or $N / r c 5^{-1-}(\mathrm{KO})$ mice were transfected with poly $(\mathrm{dA}: \mathrm{dT})(1 \mu \mathrm{g} / \mathrm{ml})(\mathrm{B})$ or poly $(\mathrm{l}: \mathrm{C})(1 \mu \mathrm{g} / \mathrm{ml})(\mathbf{C})$ for the indicated times. IFN- $\beta$ expression was determined by quantitative real-time PCR. (D) IFN- $\beta$ expression was determined in the BMMs infected as in A, by quantitative real-time PCR. Data are representative of three independent experiments (A-D) $(n=4$; mean \pm SEM in A-D).

an NLS and critically regulates the expression of MHC class II genes and other genes associated with MHC class II antigen presentation $[9,22]$. Whereas CIITA also regulates $\mathrm{MHC}$ class I gene expression in vitro, there is no reduction in MHC class I expression in CIITAdeficient mice $[9,23]$. Here, we found that MHC class II gene expression was normal in NLRC5-deficient mice, suggesting that NLRC5 and CIITA specifically regulate genes involved in MHC class I antigen presentation and MHC class II antigen presentation, respectively. CIITA associates with the MHC enhanceosome to regulate MHC class II genes $[9,24]$. Although NLRC5 binds to the promoters of MHC class I genes [11], it remains to be determined how NLRC5 regulates the expression of MHC class I genes. Whereas NLRC5 may associate with the enhanceosome utilized by CIITA, NLRC5 likely requires uncharacterized co-factors to specifically regulate the promoter activation of MHC class I genes.

In vitro studies have reported that NLRC5 negatively $[12,13]$ or positively $[10,14]$ regulates type I interferonproducing signaling pathways for host anti-viral re- sponses. NLRC5 also suppresses LPS-, TNF- or IL-1 $\beta$ induced NF- $\mathrm{BB}$-dependent gene production by directly targeting the IKK complex [12]. However, analyses of our NLRC5-deficient mice showed normal NF-kBdependent gene expression as well as normal type I interferon production in response to LPS, poly(I:C) and poly(dA:dT), which bind the receptors TLR4, RIG-I and AIM2, respectively. Our study is consistent with a recent report showing that NLRC5 is dispensable for PAMPmediated induction of NF- $\mathrm{KB}$-dependent genes or type I interferon in vivo [17].

NLRC5 regulates NLRP3 inflammasome activation in vitro [16]. Our in vivo study found that NLRC5 partly promoted NLRP3-mediated IL-1 $\beta$ maturation and contributed to innate immunity in response to LM infection by regulating neutrophil influx. However, this phenotype was not observed in one recent in vivo study [17]. One possible reason for this discrepancy might be the different targeting strategies. Our mice were generated by targeting exon 1 through exon 4 so that the first ATG codon in exon 1 was deleted, thus eliminating the poten- 
tial for a truncated NLRC5. Although NLRC5 associates with NLRP3 through its NACHT domain, the exact mechanism by which NLRC5 promotes inflammasome activation remains to be determined. Although NLRC5 partially regulated NLRP3 inflammasome activation, it did not contribute to the AIM2 ligand (poly(dA:dT))induced IL-1 $\beta$ production, which suggests that NLRC5 is not generally involved in inflammasome activation.

In conclusion, NLRC5 specifically regulates the expression of MHC class I genes and other genes associated with MHC class I antigen presentation and $\mathrm{CD} 8^{+} \mathrm{T}$ cell activation in response to intracellular bacterial infection. NLRC5 also contributes to host innate immunity by partly promoting NLRP3 inflammasome activation. This is the first study demonstrating the in vivo function of NLRC5.

\section{Materials and Methods}

\section{Mice}

Nlrc5 $5^{-1-}$ mice were generated by Shanghai Biomodel Organism Science \& Technology Development Co., Ltd. A targeting vector was constructed by replacing a $4.6-\mathrm{kb}$ fragment including the Nlrc5 open reading frame (exon 1-4) with a neomycin resistant gene cassette, and an HSV-thymidine kinase gene driven by the PGK promoter was inserted for negative selection (Supplementary information, Figure S1A). After the targeting vector was electroporated into embryonic stem cells (ESCs), G418 and ganciclovir double-resistant colonies were selected and screened by PCR. Homologous recombinant ESCs were microinjected into $\mathrm{C} 57 \mathrm{BL} / 6$ female mice, and heterozygous $\mathrm{F} 1$ progenies were intercrossed to obtain $N$ lrc $5^{-/-}$and Nlrc $5^{+/+}$mice. Nlrc $5^{-/-}$(KO) mice and littermate $N \operatorname{lrc} 5^{+/+}$mice at 6-8 weeks of age were used for experiments. The genotyping primers for $\mathrm{KO}$ allele are $5^{\prime}$-GGCCTACCCGCTTCCATTGCTC- ${ }^{\prime}$ and 5'-GTTCACCTGTTTTCCTTCCTCTCTG-3'; for wild-type allele are 5'-GTTCACCTGTTTTCCTTCCTCTCTG-3' and 5'-AAGGTACATCAAGCTCGAAGCACAG-3'. OT-1 TCR transgenic mice, specific for OVA peptide (SIINFEKL) in the context of $\mathrm{H}-2 \mathrm{~K}^{\mathrm{b}}$, were provided by Dr H Wang (Institute of Biochemistry and Cell Biology, Shanghai, China). C57BL/6 mice were purchased from Shanghai Laboratory Animal Center, Chinese Academy of Sciences. All mice were maintained in pathogen-free conditions. All animal studies were performed in compliance with the guide for the care and use of laboratory animals and are approved by the Institutional Biomedical Research Ethics Committee of the Shanghai Institutes for Biological Sciences, Chinese Academy of Sciences.

\section{Reagents}

Mouse antibodies for FACS, including anti-CD4-PE, antiCD8 $\alpha$-PE, anti-CD8 $\alpha$-FITC, anti-CD8 $\alpha-A P C$, anti-H-2K K $^{\mathrm{b}}$-FITC, anti-Ly6G-PE, anti-CD80-PE, anti-CD11b-FITC, anti-CD11c-PE, anti-IFN- $\gamma$-PE and anti-IFN- $\gamma$-FITC were from eBioscience. CFSE was from Invitrogen. Antibodies to human IL-1 $\beta, \mathrm{p}-\mathrm{I}-\mathrm{\kappa} \mathrm{B} \alpha$, p-p38 and p-JNK were from Cell Signaling Technology. Antibodies to pERK, I- $\kappa \mathrm{B} \alpha$, mouse caspase-1P10 were from Santa Cruz Biotech- nology, Inc. Antibodies to actin, M2 (Flag), caspase-1P20 were from Sigma-Aldrich (St. Louis, MO, USA). Anti-hemagglutinin (HA) (MMS-101R) was from Covance. Mouse IL-1 $\beta$ antibody, mouse IL- $1 \beta$ and IFN- $\gamma$ ELISA kits were obtained from R\&D Systems (Minneapolis, MN, USA). Anti-CD8 $\alpha$ microbeads were from Miltenyi Biotec (Auburn, CA, USA). CytoTox 96 $\square$ NonRadioactive Cytotoxicity Assay kit was from Promega. Multiplecolor flow cytometric analysis was performed using FACSAria (BD Biosciences, Franklin Lakes, NJ, USA). LPS, ATP, poly(I:C), poly(deoxyadenylicthymidylic) acid sodium salt (dA:dT), Alum (Imject Alum Adjuvant) were from Sigma-Aldrich. MSU was prepared as previously described [20].

\section{Cell culture}

HEK293 cells were maintained in DMEM supplemented with $10 \%$ (vol $/ \mathrm{vol}$ ) FBS (Hyclone), $100 \mu \mathrm{g} / \mathrm{ml}$ penicillin $\mathrm{G}$, and 100 $\mu \mathrm{g} / \mathrm{ml}$ streptomycin. HEK293 cell line stably expressing HA-ASC, HA-caspase- 1 and Flag-IL-1 $\beta$ was prepared as follows: The Nterminal HA-tagged human procaspase-1, HA-tagged human ASC or Flag-tagged human IL-1 $\beta$ was cloned into pMSCV-puromycin vector, respectively. The pMSCV plasmids and helper vectors were transfected into 293FT cells (a clonal isolate of HEK293 cells transformed with the SV40 large T antigen; Invitrogen) for viral packaging. At $60 \mathrm{~h}$ after transfection, virus was collected to infect 293T cells in the presence of polybrene $(10 \mathrm{mg} / \mathrm{ml}$; Sigma). At day 3 after infection, cells were selected for stable cell clones using puromycin, and live cells were used for experiments.

\section{Preparation of BMDCs and BMMs}

BMDCs were generated as described previously [25]. Briefly, bone marrow cells were flushed from the femurs and tibias of Nlrc $5^{-/-}$or wild-type mice and subsequently depleted of red cells with ammonium chloride. Cells were cultured at $1 \times 10^{6}$ cells per well in 24-well plates in RPMI-1640 medium containing 10\% (vol/ vol) FBS, $100 \mathrm{mg} / \mathrm{ml}$ penicillin, $100 \mathrm{mg} / \mathrm{ml}$ streptomycin, and 10 $\mathrm{ng} / \mathrm{ml}$ GM-CSF. The cultures were usually fed every 2 days by gently swirling the plates, aspirating off $75 \%$ of the medium, and adding back fresh medium with GM-CSF. On day 8, LPS (10 ng/ $\mathrm{ml}$ ) was added for DC maturation. On day 9, cells were collected for further experiments. BMMs were prepared as follows: Briefly, bone marrow cells were prepared the same as BMDCs. Cells were then cultured at $3 \times 10^{6}$ cells per well in 6-well plates in DMEM containing $10 \%$ (vol $/ \mathrm{vol}$ ) FBS, $100 \mathrm{mg} / \mathrm{ml}$ penicillin, $100 \mathrm{mg} / \mathrm{ml}$ streptomycin, and 30\% L929 conditioned medium. Nonadherent cells were removed carefully, and fresh medium was added every 2 days. On day 6, cells were collected for further experiments.

\section{Preparation of $C D 8^{+} T$ cells}

$\mathrm{CD} 8^{+} \mathrm{T}$ cells were isolated from the spleens by MACS antiCD8 $\alpha$ microbeads according to the manufacturer's instruction (Miltenyi Biotec). The purity of sorted cells in this study was consistently $>95 \%$.

\section{Listeria infections and CFU assays}

LM strain $10403 \mathrm{~s}$ or LM-expressing full-length ovalbumin protein (LM-OVA) was prepared by shaking the bacteria overnight at $37{ }^{\circ} \mathrm{C}$ in brain heart infusion (BHI) broth with $50 \mu \mathrm{g} / \mathrm{ml}$ streptomycin. In some experiments, heat-killed LM (HKLM) $\left(70{ }^{\circ} \mathrm{C}\right.$ for $1 \mathrm{~h}$ ) was used. For in vivo infections, LM were grown to mid- 
logarithmic phase, pelleted, washed, resuspended in PBS three times, and quantified by visible spectrometry readings at $600 \mathrm{~nm}$. Bacterial titer for infection was measured by plating serial dilutions on $\mathrm{BHI} /$ streptomycin agar plates. For intravenous injection, bacteria were diluted in PBS and $200 \mu 1$ was injected into mice through the lateral tail vein with the dose of $2 \times 10^{4} \mathrm{CFU}$ for all the experiments except for the experiment to check early innate immunity with a higher dose $\left(2 \times 10^{6} \mathrm{CFU}\right)$ for a short-time infection (1 day). For determination of bacterial load (CFU), mice were killed and livers or spleens were homogenized in $0.5 \%$ Triton-X solution. Serial dilutions of homogenates were plated on BHI/ streptomycin agar plates and colonies were counted after growth at $37^{\circ} \mathrm{C}$ for $24-36 \mathrm{~h}$.

\section{ELISA}

The cytokine production from the sera of Listeria-treated mice was assessed with IL- $1 \beta$ and IFN- $\gamma$ ELISA kits (R\&D Systems) according to the manufacturer's instructions. A standard curve was generated using known amounts of the respective purified recombinant mouse cytokines.

\section{RNA isolation and real-time quantitative PCR}

Total RNA was extracted from cells or mouse tissues with TRIzol reagent according to the manufacturer's instructions (Invitrogen). For cDNA synthesis, RNA was reverse-transcribed with a PrimeScript RT Reagent kit (TaKaRa), then cDNA was amplified by real-time PCR with a SYBR Premix ExTaq kit (TaKaRa) on an AbiPrism 7900 HT cycler (Applied Biosystems). The expression of target genes was normalized to expression of housekeeping gene RPL13A.

\section{Immunoprecipitation and immunoblot analysis}

The detailed protocols are followed as previously described [26].

In vitro assays of $C D 8^{+} T$ cell activation and proliferation

$\mathrm{CD}^{+} \mathrm{T}$ cells purified from the spleen of OT-1 mice were labeled with CFSE and $1 \times 10^{5}$ cells were cultured with $3 \times 10^{4} \mathrm{BM}$ DCs that had been pulsed with OVA for $1 \mathrm{~h}$ at $37{ }^{\circ} \mathrm{C}$ and washed twice prior to culture. After 3 days, supernatant was analyzed by IFN- $\gamma$ ELISA for $\mathrm{CD}^{+}$activation, while cells were harvested and labeled with anti-CD8-APC and then analyzed by flow cytometry for dilution of CFSE intensity of $\mathrm{CD}^{+}$cells for $\mathrm{CD}^{+} \mathrm{T}$ cell proliferation.

\section{Cytotoxic T lymphocytes killing (CTL) assay}

CTL assay was measured using a non-radioactive method based on release of lactate dehydrogenase (LDH) from target cells [27]. Briefly, to obtain peptide-specific CTLs, the spleen cells from naive OT-1 mice were stimulated in vitro in the presence of $10 \mathrm{nM}$ OVA peptide for 6 days. Cells were centrifuged and cultured with fresh PRMI-1640 medium, and IL-2 $(100 \mathrm{U} / \mathrm{ml})$ was added on day 3. On day 6, BMDCs were incubated with the OVA peptides $(100$ $\mathrm{pM}$ ) for $1 \mathrm{~h}$ at $37^{\circ} \mathrm{C}$. After three times of washing, $2 \times 10^{4} \mathrm{BM}$ DCs in $100 \mu \mathrm{l}$ were added to $100 \mu \mathrm{l}$ of various numbers of effector cells that had been plated in 96 round-well plates to obtain target: effector cell ratios of $1: 1.25,1: 2.5,1: 5$ or $1: 10$. After $4 \mathrm{~h}$ of incubation, the supernatant was collected to measure killing efficiency. The medium or the target cells alone were used as the low-level control (spontaneous LDH release). For the high-level control (maximum LDH release), lysate buffer was added to the target cells. The mixed cells were incubated for $4 \mathrm{~h}$ and assayed for LDH release using a cytotoxicity detection kit (LDH) (Roche Applied Science). The percentage of cell-mediated cytotoxicity was determined by the following equation: cytotoxicity $(\%)=\{[($ effector target cell mix - effector cell control) - low-level control] / (highlevel control - low-level control) $\} \times 100$.

\section{In vivo cytotoxicity assay}

The in vivo cytotoxicity assay was performed as described previously [28]. In brief, splenocytes from syngeneic mice were plused with OVA $(1 \mu \mathrm{M})$ or untreated at $37{ }^{\circ} \mathrm{C}$ for $1 \mathrm{~h}$ and then these splenocytes served as target cells, which had been labeled with cytosolic dye CFSE at a dose of $15 \mu \mathrm{M}$ for untreated splenocytes or $1.5 \mu \mathrm{M}$ for OVA-plused splenocytes. Both of the labeled cells were then mixed $(1: 1)$ and transferred i.v. with $2 \times 10^{7}$ cells in $200 \mu \mathrm{l}$ per mouse. After $15 \mathrm{~h}$ of in vivo killing, lymphocytes were isolated from the spleens and livers and analyzed by flow cytometry for target cell clearance. Target cells were differentially recognized from recipient cells based on CFSE staining and intensity. Through gating CFSE positive cells, the percentage of specific killing was calculated as follows: $100-[(\%$ peptide pulsed in LM infected / \% unpulsed in LM infected $) /(\%$ peptide pulsed in uninfected $/ \%$ unpulsed in uninfected) $] \times 100$.

\section{Statistical analysis}

Differences in the expression of genes between the groups were analyzed by the Mann-Whitney $U$ test. Two-tailed Student's $t$-test was used to analyze the differences between the groups. One-way analysis of variance was initially performed to determine whether an overall statistically significant change existed before using the two-tailed paired or unpaired Student's $t$-test. A $P$-value of $<0.05$ was considered statistically significant.

\section{Acknowledgments}

This work was supported by grants from the National Natural Science Foundation of China (30930084, 91029708 and 30871298), the National Basic Research Program of China (973 program; 2010CB529705), the Chinese Academy of Sciences (KSCX2-YW-R-146) and the Science and Technology Commission of Shanghai Municipality (10JC1416600). It was also supported by the Shanghai Hospital Science \& Technology Resource Sharing Program funded by Shanghai Shenkang Hospital Development Center (SHDC12007708).

\section{References}

1 Schroder K, Tschopp J. The inflammasomes. Cell 2010; 140:821-832.

2 Elinav E, Strowig T, Henao-Mejia J, Flavell RA. Regulation of the antimicrobial response by NLR proteins. Immunity 2011; 34:665-679.

3 Kanneganti TD. Central roles of NLRs and inflammasomes in viral infection. Nat Rev Immunol 2010; 10:688-698.

4 Kanneganti TD, Lamkanfi M, Núñez G. Intracellular NODlike receptors in host defense and disease. Immunity 2007; 27:549-559. 
5 Davis BK, Wen H, Ting JP. The inflammasome NLRs in immunity, inflammation, and associated diseases. Annu Rev Immunol 2011; 29:707-735.

6 Sorbara MT, Girardin SE. Mitochondrial ROS fuel the inflammasome. Cell Res 2011; 21:558-560.

7 Lamkanfi M, Kanneganti TD. Regulation of immune pathways by the NOD-like receptor NLRC5. Immunobiology 2011; 217:13-16.

8 Magalhaes JG, Sorbara MT, Girardin SE, Philpott DJ. What is new with Nods? Curr Opin Immunol 2010; 23:29-34.

9 Meissner TB, Li A, Kobayashi KS. NLRC5: a newly discovered MHC class I transactivator (CITA). Microbes Infect 2011 Dec 22. doi:10.1016/j.micinf.2011.12.007

10 Neerincx A, Lautz K, Menning M, et al. A role for the human nucleotide-binding domain, leucine-rich repeat-containing family member NLRC5 in antiviral responses. $\mathrm{J}$ Biol Chem 2010; 285:26223-26232.

11 Meissner TB, Li A, Biswas A, et al. NLR family member NLRC5 is a transcriptional regulator of MHC class I genes. Proc Natl Acad Sci USA 2010; 107:13794-13799.

12 Cui J, Zhu L, Xia X, et al. NLRC5 negatively regulates the NF-kappaB and type I interferon signaling pathways. Cell 2010; 141:483-496.

13 Benko S, Magalhaes JG, Philpott DJ, Girardin SE. NLRC5 limits the activation of inflammatory pathways. J Immunol 2010; 185:1681-1691.

14 Kuenzel S, Till A, Winkler M, et al. The nucleotide-binding oligomerization domain-like receptor NLRC5 is involved in IFN-dependent antiviral immune responses. J Immunol 2010; 184:1990-2000.

15 Meissner TB, Li A, Liu YJ, Gagnon E, Kobayashi KS. The nucleotide-binding domain of NLRC5 is critical for nuclear import and transactivation activity. Biochem Biophys Res Commun 2012; 418:786-791.

16 Davis BK, Roberts RA, Huang MT, et al. Cutting edge: NLRC5-dependent activation of the inflammasome. J Immunol 2010; 186:1333-1337.

17 Kumar H, Pandey S, Zou J, et al. NLRC5 deficiency does not influence cytokine induction by virus and bacteria infections. J Immunol 2010; 186:994-1000.
18 Zijlstra M, Bix M, Simister NE, et al. Beta 2-microglobulin deficient mice lack CD4-8+ cytolytic T cells. Nature 1990; 344:742-746.

19 Neefjes J, Jongsma ML, Paul P, Bakke O. Towards a systems understanding of MHC class I and MHC class II antigen presentation. Nat Rev Immunol 2011; 11:823-836.

20 Martinon F, Petrilli V, Mayor A, Tardivel A, Tschopp J. Goutassociated uric acid crystals activate the NALP3 inflammasome. Nature 2006; 440:237-241.

21 Meixenberger K, Pache F, Eitel J, et al. Listeria monocytogenes-infected human peripheral blood mononuclear cells produce IL-1beta, depending on listeriolysin O and NLRP3. $J$ Immunol 2009; 184:922-930.

22 Zika E, Ting JP. Epigenetic control of MHC-II: interplay between CIITA and histone-modifying enzymes. Curr Opin Immunol 2005; 17:58-64.

23 Chang CH, Guerder S, Hong SC, van Ewijk W, Flavell RA. Mice lacking the MHC class II transactivator (CIITA) show tissue-specific impairment of MHC class II expression. Immunity 1996; 4:167-178.

24 Masternak K, Muhlethaler-Mottet A, Villard J, et al. CIITA is a transcriptional coactivator that is recruited to MHC class II promoters by multiple synergistic interactions with an enhanceosome complex. Genes Dev 2000; 14:1156-1166.

25 Inaba K, Inaba M, Romani N, et al. Generation of large numbers of dendritic cells from mouse bone marrow cultures supplemented with granulocyte/macrophage colony-stimulating factor. J Exp Med 1992; 176:1693-1702.

26 Zhu S, Pan W, Shi P, et al. Modulation of experimental autoimmune encephalomyelitis through TRAF3-mediated suppression of interleukin 17 receptor signaling. $J$ Exp Med 2010; 207:2647-2662.

27 Hayashi T, Hideshima T, Akiyama M, et al. Ex vivo induction of multiple myeloma-specific cytotoxic T lymphocytes. Blood 2003; 102:1435-1442.

$28 \mathrm{Xu} \mathrm{H}$, Chun T, Choi HJ, Wang B, Wang CR. Impaired response to Listeria in H2-M3-deficient mice reveals a nonredundant role of MHC class Ib-specific T cells in host defense. J Exp Med 2006; 203:449-459.

(Supplementary information is linked to the online version of the paper on the Cell Research website.) 